European Symposium on Computer Aided Process Engineering - 15

L. Puigjaner and A. Espuña (Editors)

(C) 2005 Elsevier Science B.V. All rights reserved.

\title{
Integrating short-term budgeting into multi-site scheduling
}

\author{
Gonzalo Guillén, Mariana Badell, Antonio Espuña and Luis Puigjaner \\ Universitat Politècnica de Catalunya, Chemical Engineering Department, E.T.S.E.I.B., \\ Diagonal 647, E-08028, Barcelona, Spain
}

\begin{abstract}
In this work a novel approach is applied with the aim to improve the operation of supply chains with embedded multi-purpose batch chemical plants. The major contribution of this work with respect to previous approaches is that it includes a corporate financial planning model within the mathematical formulation applied for optimizing scheduling/planning decisions variables regarding the supply chain management. Such model maximizes the change in equity of the company and provides the budgetary guidelines for the planned period. This consideration exhibits two main advantages compared to the existing methodologies. In first place, it allows to check the feasibility of the resulting planning decisions from the financial viewpoint thus ensuring that the production and distribution activities to be carried out through the different nodes of the network do not spend more cash than the available one. In second place, it leads to better overall economic performance than in previous methodologies since the model properly evaluates the impact of financial expenses and earnings derived from the SC operation thus integrating production and financial corporate decisions. Such integration also makes the difference when considering the opportunity of investing the idle cash.
\end{abstract}

Keywords: agents, multi-site scheduling, financial, uncertainty.

\section{Introduction}

The concept of Supply Chain Management (SCM), which appeared in the early 90s, has recently raised a lot of interest since the opportunity of an integrated management of the $\mathrm{SC}$ can reduce the propagation of unexpected/undesirable events through the network and can influence decisively the profitability of all the members. A lot of attempts have been made to model and optimise the SC behaviour, currently existing a big amount of deterministic and stochastic derived approaches.

Most of the works reported in the literature address the SCM problem from a strategic or tactical point of view. They identify the placement of production facilities or distribution centres, the flow of materials and the inventory levels optimizing a certain performance measure, commonly cost or profit. From an operational perspective, and due to the complexity associated to the interdependencies between the production and distribution tasks of the network, the detailed scheduling of the various processes of the SC has been left to be decided locally. In this sense, Applequist et al. (1993) highlight the importance of the coordination of the activities of the different entities and specifically at the enterprise level, which requires integration of the logistics and manufacturing aspects with strategic business and financial decisions. Grossmann 
(2004) highlights also that major challenges in enterprise and supply chain optimization include development of models for strategic and tactical planning for process networks which must be eventually integrated with scheduling models. The author suggests that while very significant progress has been made, these models still lack sufficient generality despite significant advances made in this area.

A topical review of historical guidelines and approaches in integration of operative planning/scheduling and cash management modelling must take into account that budgeting models for financial control emerged earlier than operation schedules. The initial sequential approach, which focused on individual financing decisions, was later developed towards the simultaneous consideration of financial decisions. These included cash flow synchronization, financing distribution and the investment of the excess cash in marketable securities.

On the operative side, a huge number of models, especially in the last 25 years, have been developed to perform short term scheduling and longer term planning. Most of these works address scheduling/planning activities by optimizing quality or cost-related performance measures.

However, very limited works were reported on the joint financial and operative modelling. Shapiro et al. (2001) recognizes that optimization models offer an appealing framework for analyzing corporate financial decisions and constraints as well as for integrating them with supply chain decisions and constraints. Unfortunately, he also admits that relatively few corporate financial models of this type have been so far developed in the literature. If in practice the financial matters are not still integrated with operations management to support decision making, is mainly because until today scheduling/planning and budgeting modelling have been treated as separate problems and were implemented in independent environments.

\section{Multi-site planning/scheduling}

In the chemical-processing context, production planning and scheduling refers to the routine of allocating resources and equipment over time in order to execute the processing tasks required for satisfying a specific product demand and quality while fulfilling some predefined optimization criteria. Production planning implies allocation decisions over longer time scales (months), while scheduling focuses on the shorter time scale allocation thus considering those sequencing decisions that satisfy the production requirements imposed by the former.

When talking about a SC, it is important to extend the decision variables related to the plant activity to the whole network. This consideration gives rise to a muti-site scheduling/planning problem where it is necessary to decide not only the production rates of the plants and the flows of materials between sites but also the way in which such amounts of materials are manufactured (partial schedules), stored and transported through the nodes of the network.

\section{Mathematical formulation}

The proposed model divides the planning and scheduling horizon $H$ into intervals of length $H 1$ where production is planned using known as well as estimated demands 
which are provided by a forecasting tool. Moreover, the first planning period is divided into intervals of lower length $H 2$ where production is scheduled as depicted in Figure 1. The model is to be rerun every $H 1$ period as forecasts become real orders. Therefore, the results of the planning horizon beyond the first period $H 1$ will never reach execution. However, they are important to be considered when solving the scheduling horizon, because one could schedule in such period the production of materials needed in periods beyond it and keep them as inventory. At the financial side, the reschedule carried out each $H 1$ period provides a reliable forward-looking scenario aiding the synchronized financial/operative decision making.

\subsection{First stage: detailed scheduling}

In this first stage, production demands and raw materials and final product stocks are known. Here, the detailed schedules of the different sites of the SC as well as the transport decisions to be implemented through the nodes are computed. The first time period $H 1$ is divided into $t$ intervals of length $H 2$. The scheduling constraints are based on the discrete STN formulation of Shah et al. (1993), although other either continuous or discrete time scheduling formulations could be easily applied. It should be also mentioned at this point, that it is necessary to slightly modify the mass balance constraints proposed by the author for properly modelling the transport of materials through the nodes of the SC.

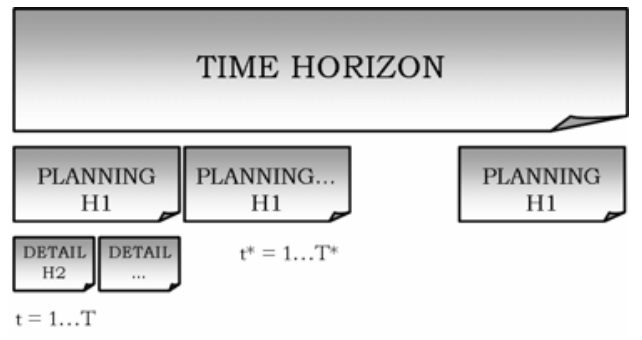

Figure 1. Structure of the model

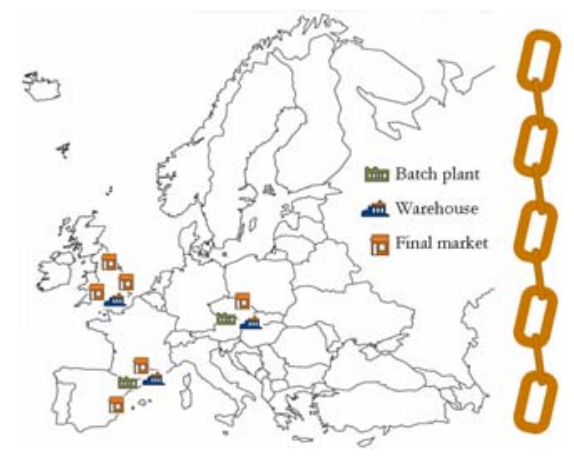

Figure 2. Case Study

\subsection{Second stage: production planning}

Here, nor the exact sequence of batches produced neither the initial and finishing times of the involved tasks are computed within every period, apart from the first one, but estimated by means of an aggregated STN representation based on the work of Maravelias and Grossmann (2004). For each task $i$, it is defined a maximum number of copies, i.e. an upper bound on the number of batches of task $i$ that can be carried out in any feasible solution. Constraint (1) is a relaxed assignment constraint which enforces that the sum of the durations of the tasks assigned to a unit does not exceed the length of each planning interval $(H I)$. Here, $I_{j}$ represents the set of tasks that can be assigned to unit $j$. In this case, it has been assumed constant processing times. The capacity limits for equipments are expressed by equation (2).

$$
\sum_{c \in C_{i}} \sum_{i \in I_{j}} p t_{i} \cdot W_{c i t} \leq H \quad \forall t
$$




$$
0 \leq B_{c i t} \leq B_{i}^{M A X} \cdot W_{c i t} \quad \forall c \in C_{i}, i, t
$$

The amount of state $s$ at the end of the time interval $t$ is calculated through constraint (3) in which it is forced to be equal to the initial amount plus the amount produced and purchased and minus the amount consumed and sold during $t$. Therefore, only assignment (1), batch size (2) and mass balance constraints (3) are included.

$S_{s t}=S_{s t-1}+\sum_{c \in C_{i}} \sum_{i \in S O_{s}} B_{c i s t}^{O}+\sum_{e}$ Purch $_{e t}^{R M}-\sum_{c \in C_{i}} \sum_{i \in S I_{s}} B_{c i s t}^{I}-$ Sales $_{s t} \quad \forall s, t$

Concerning financial matters, it should be mentioned that the cash-management constraints applied in this work have been taken from Romero et al. (2003) and consider transactions of cash due to buys or sales of marketable securities, sales of final products, payment of liabilities, the use of short-term financing sources and pledging. Nevertheless, in our formulation the change in equity achieved by the enterprise ( $\Delta$ Equity) for a given horizon of time, and not the cash withdrawn from the company as dividends, is pursued as objective aiming at the direct enhancement of the value of shareholder's interest (SHV) in the firm, which seems to be today's priority. This term can be computed as the net different between the change in assets, which include both, the current assets $(C A)$ and the fixed ones $(F A)$, and the change in liabilities, comprising the current liabilities $(C L)$ and the long-term ones $(L)$. To achieve the integration between operative and financial decisions, the production liabilities and exogenous cash at every period are calculated as a function of production planning variables. That is, the inflows of cash are determined from the sales of products assuming a known delay between the execution of the purchase and the corresponding payment, while the amount of raw materials and utilities purchased to the external suppliers are computed from the operative variables of the aggregated STN representation:

$$
\begin{aligned}
& \text { Purch }_{e t}^{\text {TOTAL }}=\sum_{c \in C_{i}} \sum_{i \in U S p_{e}} \sum_{t} W_{c i t} \cdot \alpha_{i e}+B_{c i t} \cdot \beta_{i e}+ \\
& +\sum_{s \in \text { RMSup }_{e}} \text { Purch }_{e s t}^{R M} \gamma_{s e} \cdot g_{\text {est }} \quad \forall s, t
\end{aligned}
$$

\section{Case study}

The proposed approach is applied to a case study (Figure 2), comprising two multipurpose batch plants, three warehouses and six markets. Twelve planning intervals with a length of one week each are considered. The scheduling formulation considers a time horizon of one week divided into 60 intervals of 2 hours each. The structure of the multipurpose batch plants has been taken from the case study proposed by Kondili et al. (1993). To highlight the advantages of our integrated approach, a two-step sequential scheduling-planning and budgeting approach is also applied. This situation corresponds to a typical nowadays optimized industrial routine where first operations are decided to then try to fit the finances.

The implementation in GAMS of the planning model consists of 38203 equations, 38203 continuous variables, and 6839 discrete variables. It takes $544 \mathrm{CPU}$ seconds to 
reach a solution with a $0 \%$ optimality gap on a AMD Athlon 3000 computer using the MIP solver of CPLEX (7.0). Once the scheduling-planning model is solved, the budgeting model is optimized. This model has 448 equations and 664 continuous variables. The model is solved in $0.062 \mathrm{CPU}$ seconds. On the other hand, the integrated model leads to 342485 equations, 38866 continuous variables, 6839 discrete variables and $1040 \mathrm{CPU}$ seconds to reach a solution with a $0 \%$ optimality gap on the same computer.
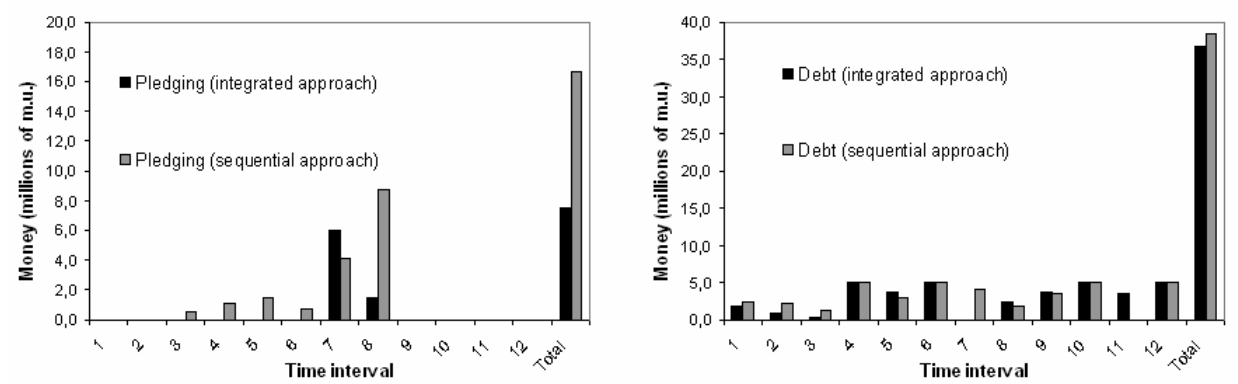

Figure 3. Total debt and amount pledged

As it can be observed in Figure 3, the solution achieved with the integrated model incurs in less debt and pledges less receivables than the sequential one. The change in equity achieved with the integrated approach is $30 \%$ higher than the sequential one (3.576.209 m.u. for the integrated model and 2.488 .312 m.u. for the sequential approach). It should be pointed out, that the case study presented here is a very specific situation where there is one product with a very high profitability in comparison with the others. Such item has a high price and consumes an expensive raw material. Given this data, the planning-scheduling model decides to fulfil the demand of this material as much as possible, what makes the budgeting model pledge receivables for purchasing the necessary raw materials. On the other hand, the integrated approach leads to lower production rates of this material, since it properly asses the financial impact derived from the operative decisions.

\section{Conclusions}

This paper has addressed the importance of integrating planning and budgeting models. This work presents a more desired measure of the effectiveness of a production plan and schedule, based on an economic performance indicator (change in equity) as an alternative to the commonly used makespan or sum of tardiness objectives. By means of a comparison using a case study, it has been shown that significant improvements are possible as compared to the use of scheduling models followed by budgeting models.

\section{Nomenclature}

$\alpha_{i e}$ cost fraction of $i$ payable to $e$

$I_{j}$ set of $j$ equipments available for task $i$

$\beta_{i e}$ cost fraction of $i$ payable to $e$

$j$ equipments

$B_{\text {cit }}$ batch size of $c$ of $i$ in $t$

$B_{c i s t}^{I}$ amount of $s$ consumed by $c$ of $i$ in $t$

$p t_{i}$ processing time of $i$

Purch $^{R M}{ }_{e s t}$ amount of $s$ purchased to $e$ in $t$ 
$B^{M A X}{ }_{i}$ maximum batch size of $i$

$B^{O}{ }_{\text {cist }}$ amount of $s$ produced by $c$ of $i$ in $t$

$c$ copies

$C_{i}$ set of copies of $i$

$e$ external suppliers

$\gamma_{\mathrm{se}}$ fraction of $s$ purchased to $e$

$t$ planning periods

$g_{\text {est }}$ price of $s$ offered by $e$ in $t$

$H$ planning period length

$i$ tasks
Purch ${ }_{\text {et }}^{\text {TOTAL }}$ total purchases payable to $e$

in $t$

$R M S u p_{e}$ set of raw materials provided by $e$

$S_{s t}$ amount of $s$ in $t$

Sales $_{s t}$ sales of $s$ in $t$

$S I_{s}$ set of states outputs of task $i$

$S O_{s}$ set of states input to task $i$

$U$ Sup $_{e}$ set of utilities provided by $e$

$W_{\text {cit }}$ binary variable (1 if $c$ of $i$ is

performed in $t, 0$ otherwise)

\section{References}

Applequist, G.E., J. F. Pekny and G. V. Reklaitis, 2000, Risk and uncertainty in managing chemical manufacturing supply chains. Comput. Chem. Eng., 53 (24), 2211-2222.

Grossmann, I. E., 2004, Challenges in the new millennium: product discovery and design, enterprise and supply chain optimization, global life cycle assessment. Comput. Chem. Eng., article in press.

Kondili, E., C. C. Pantelides and R. Sargent, 1993, A General Algorithm for Short-Term Scheduling of Batch Operations I. MILP Formulation. Comput. Chem. Eng. 17 (2), 211.

Maravelias, C. T, I. E. Grossmann, 2004, A hybrid MILP/CP decomposition approach for the continuous time scheduling of multipurpose batch plants. Comput. Chem. Eng., 28, 1921-1949.

Romero, J., M. Badell, M. Bagajewicz and L. Puigjaner, 2003, Integrating Budgeting Models into Scheduling and Planning Models for the Chemical Batch Industry. Ind. Eng. Chem. Res., 42, 6125-6134.

Shah, N., C. C. Pantelides and R. Sargent, 1993, A General Algorithm for Short-Term Scheduling of Batch Operations-II. Computational Issues. Comput. Chem. Eng., 17, 229-244.

Shapiro, J. F., 2001. Challenges of strategic supply chain planning and modelling framework. Comput. Chem. Eng., 28, 855-861.

\section{Acknowledgements}

Financial support received from the Spanish "Ministerio de Educación, Cultura y Deporte" (FPU programs) and from GICASA-D (I0353) and OCCASION (DPI200200856) projects is gratefully acknowledged. 